GLASNIK MATEMATIČKI

Vol. 40(60)(2005), $235-240$

\title{
FINITE $p$-GROUPS WITH A UNIQUENESS CONDITION FOR NON-NORMAL SUBGROUPS
}

\author{
ZVONIMIR JANKO \\ University of Heidelberg, Germany
}

\begin{abstract}
We determine up to isomorphism all finite $p$-groups $G$ which possess non-normal subgroups and each non-normal subgroup is contained in exactly one maximal subgroup of $G$. For $p=2$ this problem was essentially more difficult and we obtain in that case two new infinite families of finite 2-groups.
\end{abstract}

We consider here only finite $p$-groups and our notation is standard. It is easy to see that minimal nonabelian $p$-groups and 2-groups of maximal class have the property that each non-normal subgroup is contained in exactly one maximal subgroup. It turns out that there are two further infinite families of 2-groups which also have this property. More precisely, we shall prove the following result which gives a complete classification of such $p$-groups.

TheOREM 1. Let $G$ be a finite p-group which possesses non-normal subgroups and we assume that each non-normal subgroup of $G$ is contained in exactly one maximal subgroup. Then one of the following holds:

(a) $G$ is minimal nonabelian;

(b) $G$ is a 2-group of maximal class;

(c) $G=\langle a, b\rangle$ is a non-metacyclic 2-group, where $a^{2^{n}}=1, n \geq 3, o(b)=$ 2 or $4, a^{b}=a k, k^{2}=a^{-4},[k, a]=1, k^{b}=k^{-1}$ and we have either: (c1) $b^{2} \in\left\langle a^{2^{n-1}}, a^{2} k\right\rangle \cong E_{4}$, in which case $|G|=2^{n+2}, \Phi(G)=$ $\left\langle a^{2}\right\rangle \times\left\langle a^{2} k\right\rangle \cong C_{2^{n-1}} \times C_{2}, Z(G)=\left\langle a^{2^{n-1}}\right\rangle \times\left\langle a^{2} k\right\rangle \cong E_{4}$, and $\langle a\rangle \times\left\langle a^{2} k\right\rangle \cong C_{2^{n}} \times C_{2}$ is the unique abelian maximal subgroup of $G$, or:

2000 Mathematics Subject Classification. 20D15.

Key words and phrases. Finite p-groups, minimal nonabelian $p$-groups, 2-groups of maximal class, Hamiltonian groups. 
(c2) $b^{2} \notin\left\langle a^{2^{n-1}}, a^{2} k\right\rangle \cong E_{4}$, in which case $o(b)=4,|G|=2^{n+3}$, $\Phi(G)=\left\langle a^{2}\right\rangle \times\left\langle a^{2} k\right\rangle \times\left\langle b^{2}\right\rangle \cong C_{2^{n-1}} \times C_{2} \times C_{2}, Z(G)=\left\langle a^{2^{n-1}}\right\rangle \times$ $\left\langle a^{2} k\right\rangle \times\left\langle b^{2}\right\rangle \cong E_{8}$, and $\langle a\rangle \times\left\langle a^{2} k\right\rangle \times\left\langle b^{2}\right\rangle \cong C_{2^{n}} \times C_{2} \times C_{2}$ is the unique abelian maximal subgroup of $G$.

In any case, $G^{\prime}=\langle k\rangle \cong C_{2^{n-1}}$, a centralizes $\Phi(G)$, and $b$ inverts each element of $\Phi(G)$, and so each subgroup of $\Phi(G)$ is normal in $G$;

(d) $G=\langle a, b\rangle$ is a splitting metacyclic 2-group, where $a^{2^{n}}=b^{4}=1$, $n \geq 3, a^{b}=a^{-1} z^{\epsilon}, \epsilon=0,1, z=a^{2^{n-1}}$. Here $|G|=2^{n+2}, \Phi(G)=$ $\left\langle a^{2}\right\rangle \times\left\langle b^{2}\right\rangle \cong C_{2^{n-1}} \times C_{2}, Z(G)=\langle z\rangle \times\left\langle b^{2}\right\rangle \cong E_{4}, G^{\prime}=\left\langle a^{2}\right\rangle \cong C_{2^{n-1}}$, and $\langle a\rangle \times\left\langle b^{2}\right\rangle \cong C_{2^{n}} \times C_{2}$ is the unique abelian maximal subgroup of $G$. Since a centralizes $\Phi(G)$ and $b$ inverts each element of $\Phi(G)$, it follows that each subgroup of $\Phi(G)$ is normal in $G$.

To facilitate the proof of Theorem 1, we prove the following

Lemma 2 (Y. Berkovich). Let $G$ be a p-group, $p>2$, such that all subgroups of $\Phi(G)$ are normal in $G$. Then $\Phi(G) \leq Z(G)$.

Proof. By [1, Satz III, 7.12], $\Phi(G)$ is abelian. Suppose that $\Phi(G)$ is cyclic. Let $U / \Phi(G)$ be a subgroup of order $p$ in $G / \Phi(G)$. Assume that $U$ is nonabelian. Then $U \cong M_{p|\Phi(G)|}$ so $U=\Phi(G) \Omega_{1}(U)$, where $\Omega_{1}(U)$ is a normal subgroup of type $(p, p)$ in $G$. In that case, $\Omega_{1}(U)$ centralizes $\Phi(G)$ so $U$ is abelian, a contradiction. Let $M=\{U<G|\Phi(G)<U| U:, \Phi(G) \mid=p\}$. Then $C_{G}(\Phi(G)) \geq\langle U \mid U \in M\rangle=G$ so $\Phi(G) \leq Z(G)$.

Now let $\Phi(G)$ be noncyclic. Then $\Phi(G)=Z_{1} \times \cdots \times Z_{n}$, where $Z_{1}, \ldots, Z_{n}$ are cyclic and $n>1$. By induction on $n, \Phi\left(G / Z_{i}\right) \leq Z\left(G / Z_{i}\right)$ for all $i$. Let $f \in \Phi(G)$ and $x \in G$. Then $[f, x] \in Z_{1} \cap \cdots \cap Z_{n}=\{1\}$ so $f \in Z(G)$. It follows that $\Phi(G) \leq Z(G)$.

Proof of TheOrem 1. Let $G$ be a $p$-group which possesses non-normal subgroups and we assume that each non-normal subgroup of $G$ is contained in exactly one maximal subgroup. In particular, $G$ is nonabelian with $d(G) \geq 2$ and so each subgroup of $\Phi(G)$ must be normal in $G$. Suppose that $\Phi(G)$ is nonabelian. Then $p=2$ and $\Phi(G)$ is Hamiltonian, i.e., $\Phi(G)=Q \times E$, where $Q \cong Q_{8}$ and $\exp (E) \leq 2$. But then $E$ is normal in $G$ and $\Phi(G / E)=$ $\Phi(G) / E \cong Q_{8}$, contrary to a classical result of Burnside. Thus $\Phi(G)$ is abelian and each subgroup of $\Phi(G)$ is $G$-invariant.

If every cyclic subgroup of $G$ is normal in $G$, then every subgroup of $G$ is normal in $G$, a contradiction. Hence there is a non-normal cyclic subgroup $\langle a\rangle$ of $G$. In that case $a \notin \Phi(G)$ but $a^{p} \in \Phi(G)$ so that $\langle a\rangle \Phi(G)$ must be the unique maximal subgroup of $G$ containing $\langle a\rangle$. It follows that $d(G)=2$.

If $\Phi(G) \leq Z(G)$, then each maximal subgroup of $G$ is abelian and so $G$ is minimal nonabelian which gives the possibility (a) of our theorem.

From now on we assume that $\Phi(G) \not \leq Z(G)$. Set $G=\langle a, b\rangle$. Then $[a, b] \neq 1$ and $[a, b] \in \Phi(G)$. Therefore $\langle[a, b]\rangle$ is normal in $G$ and $G /\langle[a, b]\rangle$ 
is abelian which implies that $G^{\prime}=\langle[a, b]\rangle \neq\{1\}$. If $\left|G^{\prime}\right|=p$, then the fact $d(G)=2$ forces that $G$ would be minimal nonabelian. But then $\Phi(G) \leq Z(G)$, a contradiction. Hence $G^{\prime}$ is cyclic of order $\geq p^{2}$.

(i) First assume $p>2$. By Lemma $2, \Phi(G) \leq Z(G)$, a contradiction.

(ii) Now assume $p=2$. If $\Phi(G)$ is cyclic, then (since $\Phi(G)=\mho_{1}(G)$ ) $G$ has a cyclic subgroup of index 2 . But $\left|G^{\prime}\right| \geq 4$ and so $G$ is not isomorphic to $M_{2^{s}}, s \geq 4$, and so $G$ is of maximal class, which gives the possibility (b) of our theorem. From now on we shall assume that $\Phi(G)$ is not cyclic.

Set $G=\langle a, b\rangle, k=[a, b]$, and $\langle z\rangle=\Omega_{1}(\langle k\rangle)$ so that $G^{\prime}=\langle k\rangle, o(k) \geq 4$, and $\langle z\rangle \leq Z(G)$. Since $\left\langle a^{2}\right\rangle$ and $\left\langle b^{2}\right\rangle$ (being contained in $\Phi(G)$ ) are normal in $G$, we have $\Phi(G)=\left\langle a^{2}\right\rangle\left\langle b^{2}\right\rangle\langle k\rangle$ and so the abelian subgroup $\Phi(G)$ is a product of three cyclic subgroups which implies $d(\Phi(G))=2$ or 3 .

From $[a, b]=k$ follows $a^{-1}\left(b^{-1} a b\right)=k$ and $b^{-1}\left(a^{-1} b a\right)=k^{-1}$ and so

$$
\begin{gathered}
a^{b}=a k, \\
b^{a}=b k^{-1} .
\end{gathered}
$$

From (1) follows $\left(a^{2}\right)^{b}=\left(a^{b}\right)^{2}=(a k)^{2}=a k a k=a^{2} k^{a} k$ and so

$$
\left(a^{2}\right)^{b}=a^{2}\left(k^{a} k\right) \text {. }
$$

From (2) follows $\left(b^{2}\right)^{a}=\left(b^{a}\right)^{2}=\left(b k^{-1}\right)^{2}=b k^{-1} b k^{-1}=b^{2}\left(k^{-1}\right)^{b} k^{-1}$ and so

$$
\left(b^{2}\right)^{a}=b^{2}\left(k^{b} k\right)^{-1} .
$$

We also have

$$
a^{2}=\left(a^{2}\right)^{b^{2}}=\left(a^{2} k^{a} k\right)^{b}=a^{2} k^{a} k k^{a b} k^{b}
$$

and so

$$
k k^{a} k^{b} k^{a b}=1 .
$$

Finally, we compute (using (4))

$$
\begin{aligned}
(a b)^{2} & =a b a b=a^{2} a^{-1} b^{-1} b^{2} a b=a^{2}\left(a^{-1} b^{-1} a b\right)\left(b^{2}\right)^{a b} \\
& =a^{2} k b^{2}\left(k k^{b}\right)^{-1}=a^{2} b^{2}\left(k^{-1}\right)^{b}
\end{aligned}
$$

and so

$$
(a b)^{2}=a^{2} b^{2}\left(k^{-1}\right)^{b} .
$$

Suppose that $G / \Phi(G)$ acts faithfully on $\langle k\rangle$. In that case $o(k) \geq 2^{3}$ and we may choose the generators $a, b \in G-\Phi(G)$ so that $k^{a}=k^{-1}, k^{b}=k z$ (where $\left.\langle z\rangle=\Omega_{1}(\langle k\rangle)\right)$. Using (3) and (4) we get $\left(a^{2}\right)^{b}=a^{2}$ (and so $a^{2} \in Z(G)$ ) and $\left(b^{2}\right)^{a}=b^{2} k^{-2} z$. Since $k^{a}=k^{-1}$, we have $\langle k\rangle \cap\langle a\rangle \leq\langle z\rangle$. The subgroup $\left\langle b^{2}\right\rangle$ (being contained in $\Phi(G)$ ) is normal in $G$ and so $k^{-2} z \in\left\langle b^{2}\right\rangle$ and $k^{2} \in\left\langle b^{2}\right\rangle$ (since $z \in\left\langle k^{2}\right\rangle$ ). We have $\langle b\rangle \cap\langle k\rangle=\left\langle k^{2}\right\rangle$ since $k^{b}=k z \neq k$ and so $k \notin\langle b\rangle$. If $b^{2} \in\left\langle k^{2}\right\rangle$, then $\left(b^{2}\right)^{a}=b^{-2}$ and on the other hand $\left(b^{2}\right)^{a}=b^{2} k^{-2} z$ and so $b^{4}=k^{2} z$. But $b^{2} \in\left\langle k^{2}\right\rangle$ implies $b^{4} \in\left\langle k^{4}\right\rangle$, a contradiction. Hence $b^{2} \notin\left\langle k^{2}\right\rangle$ and so we can find an element $s \in\left\langle b^{2}\right\rangle-\langle k\rangle$ such that $s^{2}=k^{-2}$. Then 
$(s k)^{2}=s^{2} k^{2}=1$ and so $s k$ is an involution in $\Phi(G)$ which is not contained in $\langle k\rangle$ and therefore $s k \neq z$. But $(s k)^{b}=s k^{b}=(s k) z$ and so $\langle s k\rangle$ is not normal in $G$, a contradiction.

We have proved that $G / \Phi(G)$ does not act faithfully on $\langle k\rangle$. Then we can choose our generator $a \in G-\Phi(G)$ so that $k^{a}=k$. Using (3) we get $\left(a^{2}\right)^{b}=a^{2} k^{2}$ and so $1 \neq k^{2} \in\left\langle a^{2}\right\rangle$ since $\left\langle a^{2}\right\rangle$ is normal in $G$. From (5) we get $\left(k^{2}\right)^{b}=k^{-2}$. Suppose that $\left\langle k^{2}\right\rangle=\left\langle a^{2}\right\rangle$. Then we get $a^{-2}=\left(a^{2}\right)^{b}=a^{2} k^{2}$ and so $k^{2}=a^{-4}$, a contradiction. We have obtained:

$$
k^{a}=k,\left(a^{2}\right)^{b}=a^{2} k^{2},\left(k^{2}\right)^{b}=k^{-2},\{1\} \neq\left\langle k^{2}\right\rangle<\left\langle a^{2}\right\rangle, o(a)=2^{n}, n \geq 3 .
$$

Suppose that $k^{b}=k z$. Then (5) and (7) imply $k^{4}=1$ and so $k^{b}=k z=$ $k^{-1}$. It follows that we have to analyze the following three possibilities for the action of $b$ on $\langle k\rangle: k^{b}=k^{-1} z$ with $o(k) \geq 2^{3}, k^{b}=k$, and $k^{b}=k^{-1}$.

(ii1) Suppose $k^{b}=k^{-1} z$ with $o(k) \geq 2^{3}$. Then (4) gives $\left(b^{2}\right)^{a}=b^{2} z$ and so $z \in\left\langle b^{2}\right\rangle$ (since $\left\langle b^{2}\right\rangle$ is normal in $G$ ) and $\langle z\rangle\left\langle\left\langle b^{2}\right\rangle\right.$ because $b^{2} \notin Z(G)$. Since (by (7)) $\left\langle k^{2}\right\rangle<\left\langle a^{2}\right\rangle$ and $o\left(k^{2}\right) \geq 4$, it follows $o\left(a^{2}\right) \geq 2^{3}$ and

$$
\langle z\rangle=\Omega_{1}(\langle k\rangle)=\Omega_{1}(\langle a\rangle)=\Omega_{1}(\langle b\rangle) \leq Z(G) .
$$

From $o\left(a^{2}\right) \geq 2^{3}, k^{2} \in\left\langle a^{4}\right\rangle, o\left(k^{2}\right) \geq 4$, and $\left(k^{2}\right)^{b}=k^{-2}$ follows $\left(a^{2}\right)^{b}=a^{-2} z^{\epsilon}$ $(\epsilon=0,1)$ and $C_{\left\langle a^{2}\right\rangle}(b)=\langle z\rangle$ so that $\left\langle a^{2}\right\rangle \cap\left\langle b^{2}\right\rangle=\langle z\rangle$. Let $v$ be an element of order 4 in $\left\langle a^{2}\right\rangle$ so that $v^{2}=z$ and $v^{b}=v^{-1}=v z$. Let $s$ be an element of order $4 \mathrm{in}\left\langle b^{2}\right\rangle$ so that $s^{2}=z$. We have $(v s)^{2}=v^{2} s^{2}=1$ and so $v s$ is an involution in $\Phi(G)-\langle a\rangle$ but $(v s)^{b}=v^{-1} s=(v s) z$, a contradiction.

(ii2) Suppose $k^{b}=k$ so that (5) and (7) imply $k^{4}=1$ and $k^{2}=z$. Then (4) and (7) imply $\left(b^{2}\right)^{a}=b^{2} z$ and $\left(a^{2}\right)^{b}=a^{2} z$. Also, $\langle z\rangle\left\langle\left\langle a^{2}\right\rangle\right.$ and $\langle z\rangle\left\langle\left\langle b^{2}\right\rangle\right.$ since $\left\langle a^{2}\right\rangle$ and $\left\langle b^{2}\right\rangle$ are normal in $G, a^{2} \notin Z(G)$ and $b^{2} \notin Z(G)$. If $a^{2} \in\left\langle b^{2}\right\rangle$, then $a^{2} \in Z(G)$ and if $b^{2} \in\left\langle a^{2}\right\rangle$, then $b^{2} \in Z(G)$. This is a contradiction. Hence $D=\left\langle a^{2}\right\rangle \cap\left\langle b^{2}\right\rangle \geq\langle z\rangle$ and $D$ is a proper subgroup of $\left\langle a^{2}\right\rangle$ and $\left\langle b^{2}\right\rangle$. Because of the symmetry, we may assume $o(a) \geq o(b)$ so that $\left|\left\langle a^{2}\right\rangle / D\right| \geq\left|\left\langle b^{2}\right\rangle / D\right|=2^{u}, u \geq 1$. We set $\left(b^{2}\right)^{2^{u}}=d$ so that $D=\langle d\rangle$. We may choose an element $a^{\prime} \in\left\langle a^{2}\right\rangle-D$ such that $\left(a^{\prime}\right)^{2^{u}}=d^{-1}$. Then $\left(a^{\prime} b^{2}\right)^{2^{u}}=1$ and $\left\langle a^{\prime} b^{2}\right\rangle \cong C_{2^{u}}$ with $\left\langle a^{\prime} b^{2}\right\rangle \cap D=\{1\}$. On the other hand, $\left(a^{\prime} b^{2}\right)^{a}=a^{\prime}\left(b^{2}\right)^{a}=\left(a^{\prime} b^{2}\right) z$, where $z \in D$, a contradiction.

(ii3) Finally, suppose $k^{b}=k^{-1}$. From (4) follows $\left(b^{2}\right)^{a}=b^{2}$ and so $b^{2} \in Z(G)$. By $(7),\left(a^{2}\right)^{b}=a^{2} k^{2},\left\langle k^{2}\right\rangle<\left\langle a^{2}\right\rangle$, and so $o\left(a^{2}\right) \geq 4$. Also, $\left(a^{2} k\right)^{a}=a^{2} k,\left(a^{2} k\right)^{b}=\left(a^{2} k^{2}\right) k^{-1}=a^{2} k$, and so $a^{2} k \in Z(G)$.

(ii3a) First assume $k \notin\left\langle a^{2}\right\rangle$. We investigate for a moment the special case $o(k)=4$, where $k^{2}=z,\langle z\rangle=\Omega_{1}(\langle k\rangle)=\Omega_{1}(\langle a\rangle)$ and $\left(a^{2}\right)^{b}=a^{2} z$. If $o\left(a^{2}\right)>4$, then take an element $v$ of order 4 in $\left\langle a^{4}\right\rangle$ so that $v^{2}=z$ and $v^{b}=v$. In that case $(v k)^{2}=v^{2} k^{2}=1$ and so $v k$ is an involution in $\Phi(G)-\left\langle a^{2}\right\rangle$ and $(v k)^{b}=v k^{-1}=(v k) z$, a contradiction. Hence $o\left(a^{2}\right)=4$, $a^{4}=z, k^{2}=z=a^{-4},\left(a^{2}\right)^{b}=a^{2} z=a^{-2},\left\langle a^{2}, k\right\rangle$ is an abelian group of type 
$(4,2)$ acted upon invertingly by $b$, and $a^{2} k$ is a central involution in $G$. Now suppose $o(k) \geq 8$. In that case $o\left(k^{2}\right) \geq 4, k^{2} \in\left\langle a^{4}\right\rangle, o\left(a^{2}\right) \geq 8$, and $b$ inverts $\left\langle k^{2}\right\rangle$, which implies $\left(a^{2}\right)^{b}=a^{-2} z^{\epsilon}, \epsilon=0,1$. On the other hand, $\left(a^{2}\right)^{b}=a^{2} k^{2}$ and so $k^{2}=a^{-4} z^{\epsilon}$. Let $v$ be an element of order 4 in $\left\langle a^{4}\right\rangle$ so that $v^{2}=z$ and $v^{b}=v^{-1}=v z$. Then we compute:

$$
\left(a^{2} v k\right)^{2}=a^{4} z k^{2}=z^{\epsilon+1},\left(a^{2} v k\right)^{b}=a^{2} k^{2} v^{-1} k^{-1}=\left(a^{2} v k\right) z .
$$

If $\epsilon=1$, then $a^{2} v k$ is an involution in $\Phi(G)-\left\langle a^{2}\right\rangle$ and $\left\langle a^{2} v k\right\rangle$ is not normal in $G$. Thus, $\epsilon=0,\left(a^{2}\right)^{b}=a^{-2}, k^{2}=a^{-4}, a^{2} k$ is an involution in $\Phi(G)-\left\langle a^{2}\right\rangle$ and $b$ inverts each element of $\left\langle a^{2}, k\right\rangle=\left\langle a^{2}\right\rangle \times\left\langle a^{2} k\right\rangle$, where $a^{2} k \in Z(G)$.

We have proved that in any case $k^{2}=a^{-4}, o\left(a^{2}\right) \geq 4, o(k) \geq 4$, and $b$ inverts each element of the abelian group $\left\langle a^{2}, k\right\rangle=\left\langle a^{2}\right\rangle \times\left\langle a^{2} k\right\rangle$, where $a^{2} k$ is an involution contained in $Z(G)$.

It remains to determine $b^{2} \in Z(G)$. Suppose $o\left(b^{2}\right) \geq 4$ and let $\langle s\rangle$ be a cyclic subgroup of order 4 in $\left\langle b^{2}\right\rangle$ so that $s \in Z(G)$. Obviously, $s \notin\left\langle a^{2}, k\right\rangle$ since $Z(G) \cap\left\langle a^{2}, k\right\rangle=\langle z\rangle \times\left\langle a^{2} k\right\rangle \cong E_{4}$. Let $v$ be an element of order 4 in $\left\langle a^{2}\right\rangle$ so that $v^{2}=z$ and $v^{b}=v^{-1}=v z$. We have:

$$
(v s)^{b}=v^{-1} s=(v s) z \text { and }(v s)^{2}=v^{2} s^{2}=z s^{2} .
$$

If $s^{2}=z$, then $v s$ is an involution in $\Phi(G)-\left\langle a^{2}, k\right\rangle$ and $v s \notin Z(G)$, a contradiction. Hence $s^{2} \neq z$ so that $\langle v, s\rangle=\langle v\rangle \times\langle s\rangle \cong C_{4} \times C_{4}$. But $(v s)^{b}=(v s) z,(v s)^{2}=z s^{2} \neq z$, and so $\langle v s\rangle$ is not normal in $G$, a contradiction. It follows that $o\left(b^{2}\right) \leq 2$. Hence we have either $b^{2} \in\left\langle z, a^{2} k\right\rangle, \Phi(G)=\left\langle a^{2}, k\right\rangle=$ $\left\langle a^{2}\right\rangle \times\left\langle a^{2} k\right\rangle$, and we have obtained the possibility (c1) of our theorem or $b^{2}$ is an involution in $\Phi(G)-\left\langle a^{2}, k\right\rangle, \Phi(G)=\left\langle a^{2}\right\rangle \times\left\langle a^{2} k\right\rangle \times\left\langle b^{2}\right\rangle$, and we have obtained the possibility (c2) of our theorem. Note that in both cases $a$ centralizes $\Phi(G)$ and $b$ inverts each element of $\Phi(G)$.

(ii3b) We assume $k \in\left\langle a^{2}\right\rangle$. Since $o(k) \geq 4, k^{b}=k^{-1},\langle a\rangle$ is normal in $G, o(a) \geq 8$, and $b$ induces on $\langle a\rangle$ an automorphism of order 2, we get $a^{b}=a^{-1} z^{\epsilon}, \epsilon=0,1$, where $\langle z\rangle=\Omega_{1}(\langle a\rangle)=\Omega_{1}(\langle k\rangle)$. On the other hand, (1) gives $a^{b}=a k$ and so $k=a^{-2} z^{\epsilon}$ which gives $G^{\prime}=\langle k\rangle=\left\langle a^{2}\right\rangle \cong C_{2^{n-1}}$, where $o(a)=2^{n}, n \geq 3$, and $z=a^{2^{n-1}}$.

Since $\Phi(G)=\left\langle a^{2}, b^{2}\right\rangle$ and $\Phi(G)$ is noncyclic, we have $b^{2} \notin\left\langle a^{2}\right\rangle$ and we know that $b^{2} \in Z(G)$. Suppose $o\left(b^{2}\right) \geq 4$ and let $s$ be an element of order 4 in $\left\langle b^{2}\right\rangle$. Let $v$ be an element of order 4 in $\left\langle a^{2}\right\rangle$ so that $v^{2}=z$ and $v^{b}=v^{-1}=v z$. Then

$$
(v s)^{b}=v^{-1} s=(v s) z \text { and }(v s)^{2}=v^{2} s^{2}=z s^{2} .
$$

If $s^{2}=z$, then $v s$ is an involution in $\Phi(G)-\left\langle a^{2}\right\rangle$ and $v s \notin Z(G)$, a contradiction. Hence $s^{2} \neq z$ so that $\langle v, s\rangle=\langle v\rangle \times\langle s\rangle \cong C_{4} \times C_{4}$. But $\langle v s\rangle$ is not normal in $G$, a contradiction. Hence $b^{2}$ is an involution in $\Phi(G)-\left\langle a^{2}\right\rangle$ and so $\Phi(G)=\left\langle a^{2}\right\rangle \times\left\langle b^{2}\right\rangle \cong C_{2^{n-1}} \times C_{2}$ and $Z(G)=\langle z\rangle \times\left\langle b^{2}\right\rangle \cong E_{4}$. Also note that $a$ centralizes $\Phi(G)$ and $b$ inverts each element of $\Phi(G)$. We have obtained the possibility (d) of our theorem. 


\section{REFERENCES}

[1] B. Huppert, Endliche Gruppen 1, Springer, Berlin, (1967).

Z. Janko

Mathematical Institute, University of Heidelberg,

69120 Heidelberg, Germany

E-mail: janko@mathi.uni-heidelberg.de

Received: 31.1.2005. 